\title{
New viral gastrointestinal agents associated with paediatrics illness
}

\author{
Sara Giordana Rimoldi', Rosaria Bartolone', Nunzia Calvagna', Dario Dilillo, \\ Maria Rita Gismondo', Alessandra Lombardi', Nadia Zanchetta', Gian Vincenzo Zuccotti' \\ 'Unità Operativa di Microbiologia AO Polo Universitario “L.Sacco", Milano, Italy \\ ${ }^{2}$ Clinica Pediatrica "L.Sacco", Milano, Italy
}

Key Words: Noroviruses, Bocaviruses, gastroenteritis

Infezioni gastroenteriche sostenute da patogeni emergenti in età pediatrica

\section{SUMMARY}

\section{Objective}

Aim of our study was investigate the presence of enteric viruses like Norovirus, Bocavirus and Rotavirus in hospitalized children belong to Paediatrics Clinics of L. Sacco Hospital.

Material and methods

I50 faecal samples were investigated from January 2008- 2009, by PCR real time for Norovirus and Bocavirus; Rotavirus was detected by enzyme immunoassay.

\section{Results}

We obtain a positive result on total samples in percentage of $36 \%$ : Rotaviruses are estimated to cause more than $20 \%$ of gastroenteritis cases; Noroviruses $7.3 \%$ cases; Bocaviruses $6.6 \%$ cases. Rotaviruses infections occurred in children I-4 years of age and for 2 cases at 7 and 13 years old. Illness typically happened in the cooler months from January to March even if there were few positive cases in September/October. Noroviruses and Bocaviruses infections occurred in children I-2 years of age and their seasonality were respectively from September to December and from January to March for Bocaviruses.

\section{Conclusions}

The present results point out the importance to detect new viral gastrointestinal agents associated with paediatric illness.

\section{INTRODUZIONE}

La diarrea intesa come aumento della frequenza, del volume e della fluidità delle evacuazioni è un sintomo appartenente a varie condizioni morbose di cui sono noti milioni di casi/anno, tanto da rappresentare un grosso problema di sanità pubblica, per le visite specialistiche necessarie e i ricoveri ospedalieri conseguenti.

La causa infettiva di diarrea nota come gastroenterite (GE) è tra i maggiori responsabili di morbosità e mortalità nel mondo in tutte le fasce d'età, anche se sono più colpite quelle agli estremi della vita, tanto da risultare un grosso problema nosocomiale nei reparti pediatrici e geriatrici (1).

In Italia, nonostante l'elevata morbilità di tale patologia, manca di un sistema organico di sorveglianza nazionale delle patologie acute del tratto gastroenterico in età infantile, non permettendo un'accurata conoscenza dell'epidemiologia italiana, tanto che gastroenteriti non adeguatamente diagnosticate rimangono di causa sconosciuta.

Dalla letteratura emerge che le GE infantili endemiche siano dovute per il $70 \%$ ad agenti virali, un $30 \%$ sostenuta da batteri e solo 3\% siano di natura parassitaria.

I virus maggiormente responsabili di GE sono Rotavirus, Adenovirus, Norovirus e Astrovirus. Possono dare anche sintomatologia gastroenterica anche gli Enterovirus specialmente con coxachie ed Echovirus; Bocavirus, identificati inizialmente come virus espiratori che possono dare sintomatologia gastroenterica (2).

Caratteristica che accomuna tali patogeni è la loro struttura a RNA, eccezion fatta per Bocavirus e Adenovirus che sono a DNA $(3,4)$.

Il meccanismo patogenetico che porta a malattia è

\section{Corresponding author: Sara Giordana Rimoldi}

Ospedale "L. Sacco" - Laboratorio Microbiologia

Via G.B. Grassi, 74 - 20I55, Milano, Italy

E-mail: rimoldi.sara@hsacco.it 
per tutti dovuto all'infezione da parte dei virus citati degli enterociti dell'epitelio villoso del piccolo intestino, con conseguente replicazione nelle cellule epiteliali, distruzione delle stesse e trasudazione dei liquidi e dei Sali nel lume intestinale. Il danno morfologico conseguente porta all'emissione di feci non formate per più volte nell'arco della giornata. Infatti, anche se la maggior parte delle infezioni gastroenteriche virali è asintomati$\mathrm{ca}$, in quelle sintomatiche la diarrea è il sintomo più frequente. La durata della stessa può variare da patogeno a patogeno: 5/7 giorni per Rotavirus, fino a due settimane nel caso di Adenovirus.

\section{MATERIALI E METODI}

Per questo studio, condotto nel periodo compreso tra Gennaio 2008 e Gennaio 2009, sono stati raccolti 150 campioni fecali di pazienti pediatrici provenienti dalla Clinica Pediatrica dell’Azienda Ospedaliera Polo Universitario L.Sacco, dove erano stati ricoverati per gastroenterite acuta.

I pazienti selezionati presentavano una sintomatologia propriamente gastroenterica, con diarrea o dissenteria profusa e vomito della durata di più giorni, febbre, disidratazione. Tutti i pazienti arruolati sono stati valutati mediante lo Score di classificazione della severità clinica della gastroenterite in lieve, moderata e grave secondo Ruska e Vesikari (5).

Tale score di valutazione della gravità delle diarree permette di assegnare un punteggio sulla base di parametri clinici che riguardano la durata della diarrea (giorni), le scariche diarroiche per die, il numero dei giorni di vomito, febbre, disidratazione ed eventuale terapia idratante osservata per os o mediante ospedalizzazione

I campioni raccolti venivano testati dapprima mediante coprocoltura per la ricerca di Salmonella spp., Shigella spp., Campilobacter e Yersinia; successivamente venivano testati per Rotavirus mediante mediante la ricerca dell'anti- gene specifico ( Meridian).

Bocavirus e Norovirus sono stati testati mediante Real time PCR(Argene, Francia)

\section{RISULTATI}

I dati ottenuti dal presente studio eseguito su pazienti pediatrici hanno documentato un ruolo importante dei patogeni virali emergenti testati nel sostegno delle infezioni gastrointestinali. Come è possibile osservare in Tabella 2, da una valutazione condotta su 150 campioni fecali pervenuti al Laboratorio di Microbiologia e Virologia da pazienti pediatrici ricoverati presso la Clinica Pediatrica dell'A.O e Polo Universitario L.Sacco tra Gennaio 2008 e Gennaio 2009, è emerso che 32 campioni testati erano positivi per Rotavirus; (21.3\%); 11 positivi per Norovirus (7.3\%); 10 positivi per Bocavirus (6.5\%). La percentuale di campioni risultati positivi per virus è stata del $36 \%$; in soli due casi i campioni erano postivi per batteri, rispettivamente $C$ difficile e per Salmonella (1.3\%)

Nel Grafico 1, si mostra la distribuzione per età dei pazienti positivi per Norovirus (colonna blu) e Bocavirus (colonna rossa). Un solo paziente con test per Bocavirus positivo aveva un'età inferiore al mese di vita, due pazienti con 3 mesi di vita, 5 pazienti avevano 9 mesi ; non si sono avute positività per Bocavirus a 2, 5, 12, 18 e 24 mesi.

Il test per Norovirus è risultato positivo per 3 pazienti sotto il mese di vita, 2 pazienti di 2 mesi, 3 pazienti rispettivamente di 5, 12,18 e 24 mesi. Nessun paziente è risultato avere una positività per Norovirus all'età di tre mesi.

Tabella 2. Frequenza e distribuzione delle infezioni virali in pazienti pediatrici ricoverati per gastroenterite nel periodo Gennaio2008-Gennaio 2009

\begin{tabular}{lccc}
\hline Microrganismo & Norovirus & Bocavirus & Rotavirus \\
\hline Frequenza[\%] & $7,3 \%$ & $6,5 \%$ & $21,3 \%$ \\
\hline Distribuzione & $11(150)$ & $10(150)$ & $32(150)$ \\
\hline
\end{tabular}

Tabella I.Caratteristiche dei principali agenti virali implicati nelle infezioni gastroenteriche

\begin{tabular}{|c|c|c|c|c|c|}
\hline Virus & Segni e sintomi & Incubazione & Durata & $\begin{array}{c}\text { Modalità } \\
\text { d'infezione }\end{array}$ & Stagionalità \\
\hline Astrovirus & $\begin{array}{l}\text { Sindrome simile ad una } \\
\text { lieve infezione da Rotavirus }\end{array}$ & I-3 giorni & 2-3 giorni & Acqua, cibo & $\begin{array}{c}\text { Mesi } \\
\text { invernali }\end{array}$ \\
\hline Adenovirus & Diarrea & 8-10 giorni & $\begin{array}{c}\mathrm{I}-2 \\
\text { settimane }\end{array}$ & $\begin{array}{c}\text { Trasmissione } \\
\text { oro-fecale }\end{array}$ & $\begin{array}{c}\text { Prevalenza nei } \\
\text { mesi estivi } \\
\end{array}$ \\
\hline Bocavirus & $\begin{array}{l}\text { Sintomi respiratori e } \\
\text { gastroeintestinali }\end{array}$ & I-4 giorni & 2-I0 giorni & $\begin{array}{c}\text { Trasmissione } \\
\text { aerogena }\end{array}$ & $\begin{array}{c}\text { Mesi } \\
\text { invernali } \\
\end{array}$ \\
\hline Rotavirus & $\begin{array}{l}\text { Diarrea acquosa, vomito, } \\
\text { febbre }>39{ }^{\circ} \mathrm{C}\end{array}$ & I-3 giorni & 5-7 giorni & $\begin{array}{c}\text { Trasmissione } \\
\text { oro-fecale }\end{array}$ & $\begin{array}{l}\text { Novembre } \\
\text { - Marzo }\end{array}$ \\
\hline Norovirus & $\begin{array}{l}\text { Vomito ad inizio acuto, } \\
\text { diarrea, crampi addominali, } \\
\text { occasionalmente febbre, } \\
\text { mialgie e cefalea }\end{array}$ & $12-17$ ore & I-2 giorni & $\begin{array}{l}\text { Cibi, } \\
\text { superfici infette, } \\
\text { contatto } \\
\text { persona-persona }\end{array}$ & $\begin{array}{c}\text { Mesi } \\
\text { invernali }\end{array}$ \\
\hline
\end{tabular}


Nel Grafico 2 sono state, invece, riportate le sole distribuzioni per età dei pazienti positivi al test per Rotavirus: tali pazienti avevano un'età maggiore rispetto a quelli precedentemente mostrati nel Grafico 1 (14 casi avevano un anno d'età; 7 avevano 2 anni d'età, 5 avevano 3 anni d'età, 2 avevano 4 anni e a uno solo caso rispettivamente 7 e 13 anni). Non si sono riscontrate positività all'età di 5, 6, 8, 9, 10, 11 e 12 anni.

È stata quindi eseguita una valutazione della stagionalità di Rotavirus, Norovirus e Bocavirus. La positività massima è stata raggiunta tra Gennaio e Febbraio (Grafico 3); nei mesi di Marzo, Aprile e Maggio c'è stata una diminuzione e quindi una ripresa lieve a Settembre ed Ottobre.

Nel Grafico 4 è stata rappresentata la stagionalità complessiva di tutti i patogeni considerati.

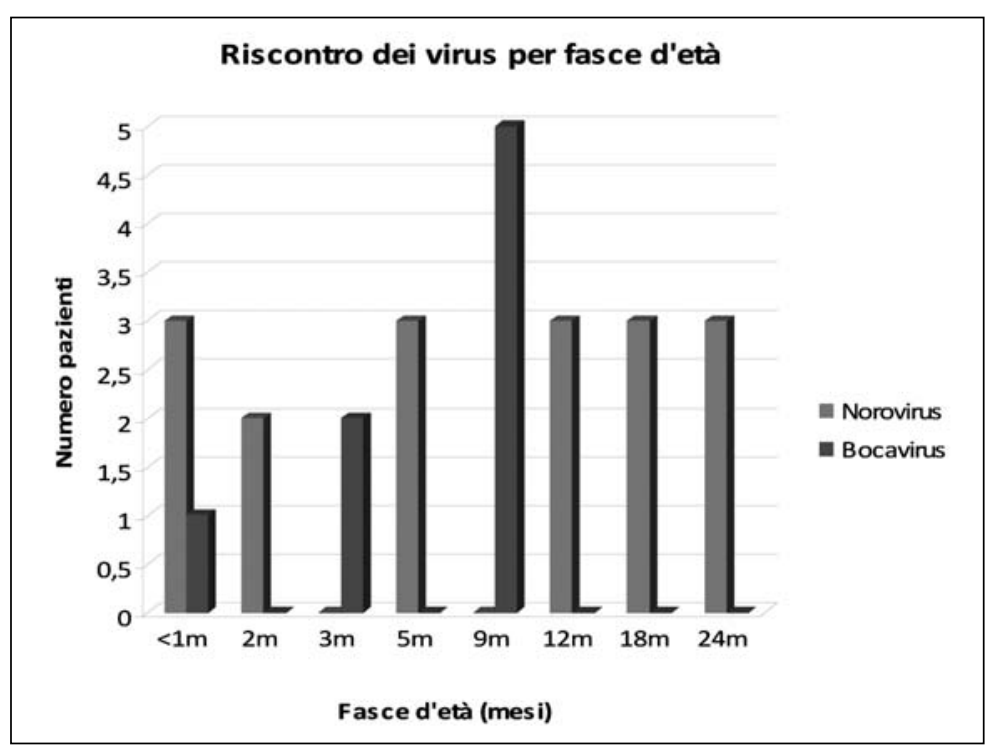

Grafico I. Valutazione per fasce d'età dei pazienti al test per Norovirus e Bocavirus

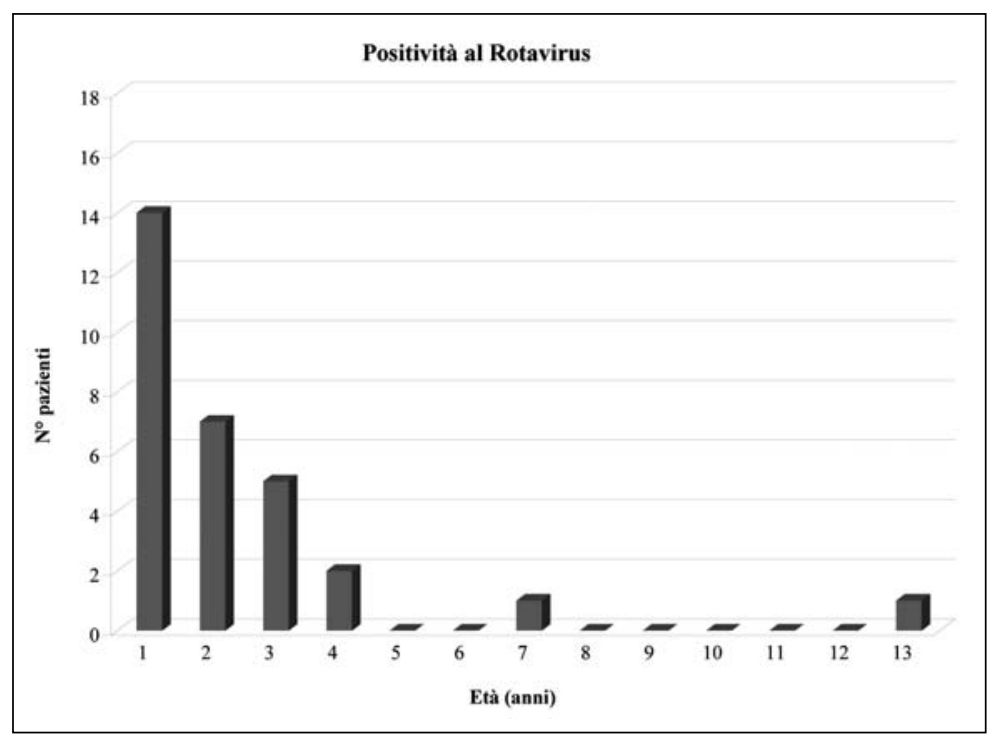

Grafico 2. Valutazione per anni d'età dei pazienti risultati positivi al test per Rotavirus
All'interno delle positività per i rispettivi patogeni considerati sono state riscontrate delle coinfezioni: 7 pazienti dei 32 positivi a Rotavirus risultavano positivi sia a Norovirus (1 caso) che a Bocavirus ( 6 casi).

\section{DISCUSSIONE}

Diversi sono gli agenti eziologici responsabili di gastroenterite virale (43) e scarsi sono i sistemi di sorveglianza in grado di documentare nel nostro Paese l'epidemiologia delle infezioni gastroenteriche in età pediatrica. Notevole interesse nella letteratura internazionale stanno acquisendo i Norovirus e Bocavirus (44), considerati patogeni emergenti nelle infezioni gastroenteriche.

Il nostro studio, che ha coinvolto attivamente l'U.O Complessa di Microbiologia e Virologia e la Clinica Pediatrica dell'A.O. Polo Universitario L Sacco, prevedeva l' ampliamento dello screening del pannello dei virus enterici coinvolti nelle gastroenteriti pediatriche, permettendo così di rilevare un'alta incidenza di tali agenti eziologici nei pazienti di età inferiore ai due anni, anche in assenza di epidemia (45). I dati da noi osservati hanno reso possibile la rilevazione di casi sporadici di infezione da NoV in un periodo di osservazione compreso tra Settembre-Dicembre 2008 con 10 casi positivi riscontrati e supportati da sintomatologia propriamente gastroenterica, non osservata invece nell'inverno precedente.

La stagionalità osservata è in linea con quanto osservato in letteratura (46).

Significativa è la percentuale di coinfezioni sostenute da Rotavirus vs Norovirus e Rotavirus vs Bocavirus (6). Per quanto la tendenza di pediatri e medici di base a fronte di diarrea e dissenteria sia di attendere la risoluzione della malattia (se il patogeno identificato con test batteriologici non rientra tra quelli maggiormente aggressivi e invasivi, come Salmonella spp., Campylobacter spp., Shigella spp., Escherichia coli), appare importante poter inserire nella routine di laboratorio lo screening di patogeni quali quelli da noi indagati al fine di poter assegnare un agente eziologico a tutte quelle GE di causa sconosciuta.

Tale informazione è utile sia per una valutazione epidemiologica locale dei patogeni circolanti, sia per evitare l'uso di rimedi farmacologici 


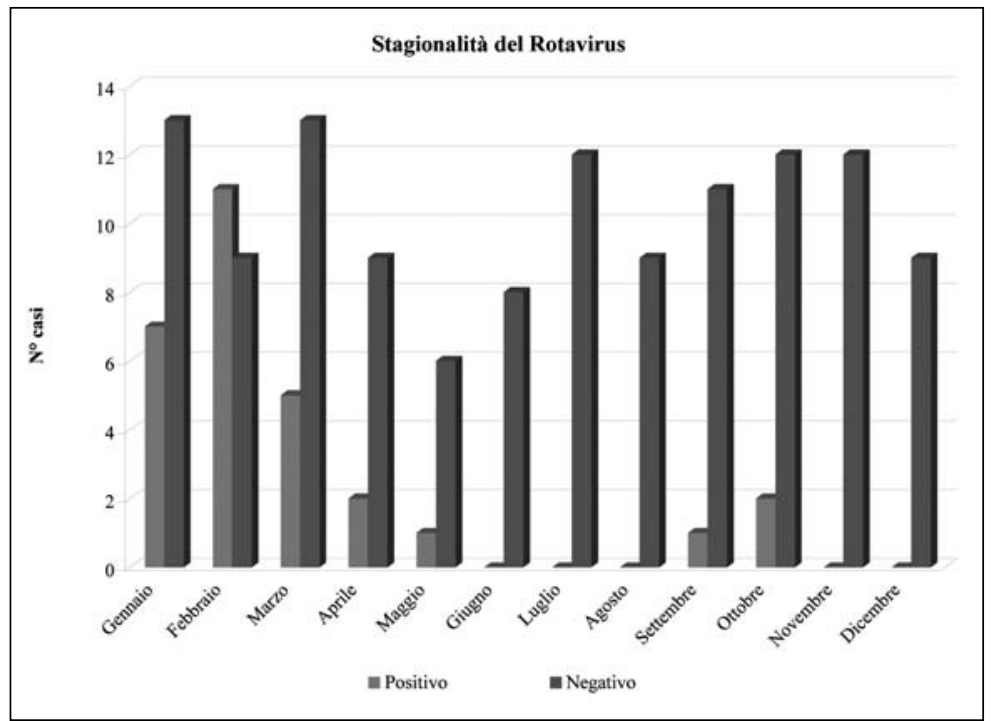

Grafico 3. Valutazione della stagionalità del Rotavirus osservata nell'anno 2008 costosi e inutili, quali gli antibiotici, di fronte ad infezioni di origine virale (48-50).

La determinazione in tempi brevi, mediante tecnologie diagnostiche rapide, di patogeni alert da parte del Laboratorio di Microbiologia, opportunamente comunicati al clinico di reparto che isola il paziente, limitano l'insorgenza di flare epidemici difficili da controllare e ampiamente riportati in letteratura, soprattutto nelle strutture residenziali sanitario assistenziali. Documentata da altri gruppi di ricerca $(6,7)$ la presenza di una positività crociata tra Rotavirus e Norovirus; nulla si sa ancora di preciso sul significato clinico tra la coinfezione Rotavirus e Bocavirus (8).

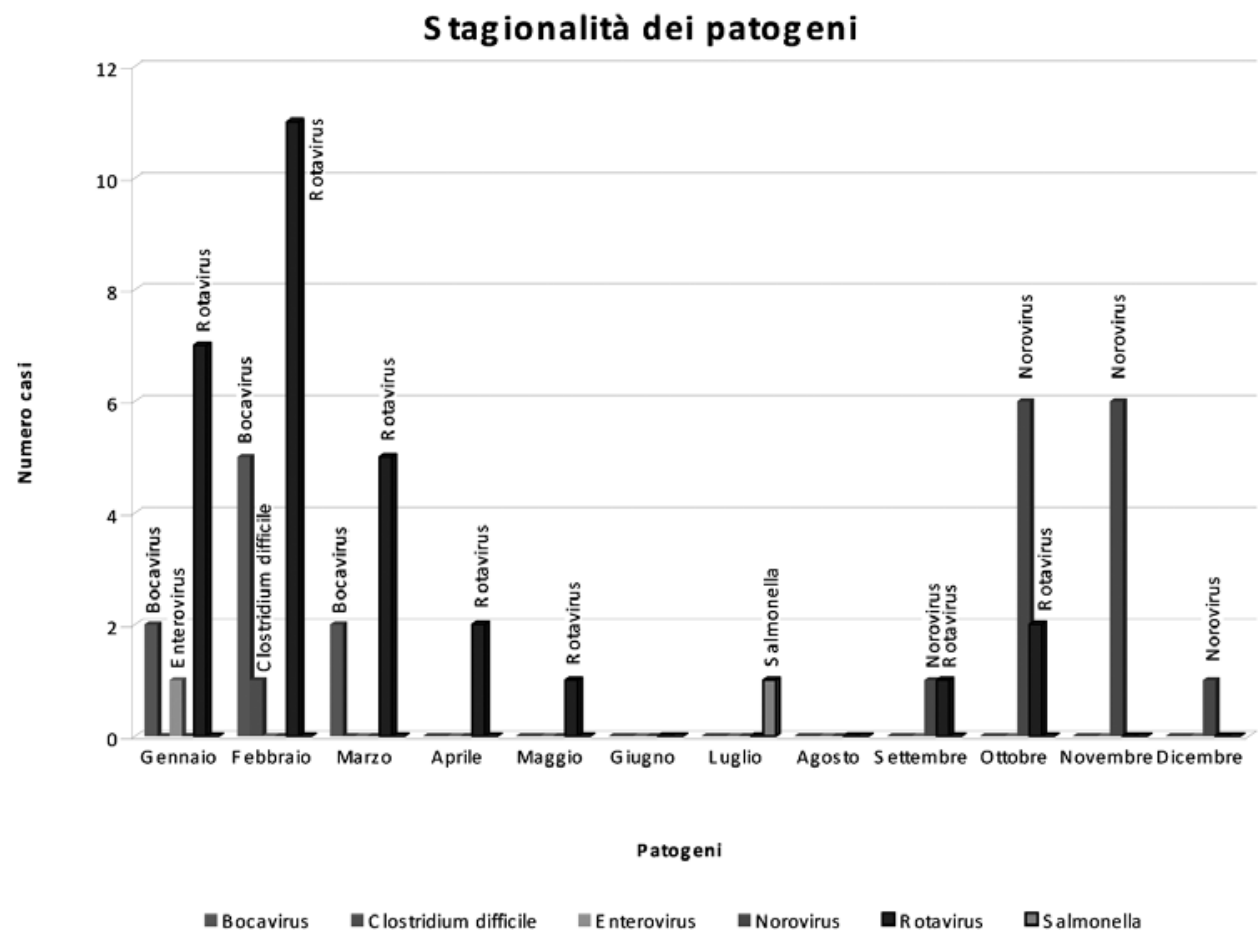

Grafico 4. Valutazione della stagionalità dei patogeni indagati osservata nell'anno 2008

\section{BIBLIOGRAFIA}

1. Albuquerque MC, et al. Human Bocavirus infection in children with gastroenteritis, Brazil. Emerg Infect Dis. 2007 Nov; 13(11): 1756-8

2. Baez NR, et al. Astrovirus, Adenovirus and Rotavirus in hospitalized children:prevalence and association with gastroenteritis. $\mathrm{J}$ of pediatric gastroenterology and nutrition; 35(1), 2002

3. Campe H, et al. Role of Human Bocavirus infections in outbreaks of gastroenteritis. J clin virol. 2008; 43(3): 340-342

4. Colomba C, et al. Emerging Infectious Diseases; Norovirus and Gastroenteritis in hospitalized children, Italy. 2007 (13): 1389-1391
5. Esposito S, et al . Impact of Human Bocavirus on children and their families J Clinical Microbiology 2008;46(4):1337-1342

6. Koh $\mathrm{H}$, et al Coinfection of viral agents in Korean childrea with acute watery diarrhea.J Korean Med Sci.2008; 23:937-940

7. Maggi F, et al. Human Bocavirus in Italian patients with respiratory diseases. J Clin Virology 2007; 38: 321-325

8. Ruska $\mathrm{T}$, Versikari T. Rotavirus disease in finnish children: use of numerical scores for clinical severity of diarrhoeal episodes. Scand J Infect Dis, 1990; 22:259-267

9. Santos R, et al. Astrovirus infection in children living in the Central West region of Brasil, 2007; 102(2): 2009-213 